\title{
A BP Neural Network Method for Grade Classification of Loose Damage in Semirigid Pavement Bases
}

\author{
Bei Zhang $\left(\mathbb{D}\right.$, Jianyang Liu, Yanhui Zhong $\mathbb{D}^{D}$, Xiaolong Li $\mathbb{D}$, Meimei Hao $\mathbb{D}$, Xiao Li, \\ Xu Zhang, and Xiaoliang Wang
}

College of Water Conservancy and Engineering, Zhengzhou University, Zhengzhou 450001, China

Correspondence should be addressed to Yanhui Zhong; zhong_yanhui@163.com and Xiaolong Li; wennuandeshang@ hotmail.com

Received 9 December 2020; Revised 7 January 2021; Accepted 28 January 2021; Published 10 February 2021

Academic Editor: Di Wang

Copyright (c) 2021 Bei Zhang et al. This is an open access article distributed under the Creative Commons Attribution License, which permits unrestricted use, distribution, and reproduction in any medium, provided the original work is properly cited.

This study aims to address the problem that loose damage of the pavement base course cannot currently be quantitatively identified, and thus the classification and recognition of the extent of looseness mainly rely on empirical judgments. Based on the finite-difference time-domain (FDTD) method, a backpropagation (BP) neural network identification method for loose damage of a semirigid base is presented. The FDTD method is used to simulate a semirigid base road model numerically with different degrees of looseness, and the eigenvalue parameters for recognition of the presence and extent of the looseness of the base layer are obtained. Then, a BP neural network identification method is used to classify and identify the loose damage of the base course. The results show that the classification and recognition of simulated electromagnetic waves have an accuracy of over $90 \%$; the classification and recognition of radar data from an actual project have a recognition accuracy of over $80 \%$. The good agreement between the classification and recognition results for the simulated data and measured data verifies the feasibility of the classification and recognition method, which can provide a new method for the use of ground-penetrating radar to detect loose damage and the extent of looseness in the base.

\section{Introduction}

A semirigid base has a high-capacity strength, good integrity, low cost, local materials, and excellent mechanical and road performance; therefore, these are widely used in road engineering for pavement bases and subbases [1]. Accordingly, more than $90 \%$ of the expressways under construction or completed in China are composed of semirigid base asphalt pavement $[2,3]$. The popularization and application of semirigid bases has played an important role in the construction of expressways and economic development in China. According to the Chinese road construction specifications, the service life of expressways and first-class highways is fifteen years, while that of secondary roads is twelve years. However, owing to the substandard design and construction of some roads, as well as the rapid increase in heavy-load traffic and traffic volumes, many roads exhibit damage comprising different degrees of looseness before their service life is reached. Under the influence of loads and environmental changes, structural shrinkage will occur in loose parts of the semirigid base, which will cause crack damage and eventually lead to reflection cracks in the pavement. The cracked pavement will directly affect the quality and service life of the road [4-6]. Therefore, it is necessary to detect damage in the semirigid base before the pavement is severely damaged. In the early stage of road detection, core sampling drilling is often used $[7,8]$; however, core sampling drilling is destructive to the road structure. As a result, it is difficult to carry out a large number of sampling inspections, and it is difficult to reflect the overall quality of the road structure using only a small number of results. As a nondestructive testing method, ground-penetrating radar (GPR) [9-11] has the advantages of portability, high detection efficiency, high resolution, and strong anti-interference ability compared with ultrasonic testing $[12,13]$, core sampling drilling, and infrared imaging 
[14]. Chahine et al. proposed an algorithm for ultrathin layer thickness estimation based on independent component analysis. The experimental results using simulation data showed that the relative error of the ultrathin layer thickness estimation was $0.9 \%$ when $B \Delta \tau=0.45$ (where $B$ is the bandwidth of the radar system, and $\Delta \tau$ is the minimum time delay that the system can identify); the signal-to-noise ratio was $0.35 \mathrm{Db}$ [15]. Cardarelli used GPR to detect the loose area of a tunnel in combination with the seismic tomography method [16]. Karem et al. used GPR to detect buried objects, and the scale-invariant feature transform (SIFT) algorithm was applied to extract the features of each location. The results showed that the introduction of the SIFT algorithm improved the detection accuracy [17]. Tong et al. applied convolutional neural networks (CNNs) to recognize and locate concealed cracks automatically based on GPR images $[18,19]$. Cubero-Fernandez et al. proposed an effective method for both pavement crack detection and classification [20]. Soola et al. combined GPR technology with infrared images to detect the depth characteristics and origin of asphalt pavement cracks. Although this detection method can determine the location and depth of subgrade cracks, its measurement accuracy is relatively poor [21]. Ditommaso et al. used GPR to analyze the problem of water pipe rupture in road structures and adopted a new operation method, the intensive matrix, to locate underground plastic drainage pipes while maintaining the original state and environmental characteristics of the system. The research results showed that the multiagent test could accurately locate the position of the plastic pipe [22]. Xu et al. used the GPR with $300 \mathrm{MHz}$ shielded antenna to detect the cracks in the sand gravel subgrade. Through the radar image, the crack location and crack length can be accurately located, and the crack length can be calculated [23]. Zhang et al. also studied GPR technology and proposed a set of road damage identification methods based on GPR and a falling weight deflectometer. This method could accurately determine the location of road damage through nondestructive testing and evaluate various indicators of the road state $[24,25]$.

To date, GPR has been widely used to study road voids, thickness, cracks, etc.; however, little research has been conducted on the loose damage of the base. Loose damage can only be analyzed qualitatively, and the extent of the looseness of the base cannot be classified. Therefore, in this study, starting from the detection and determination of the porosity of areas subject to loose damage, the finite difference time-domain (FDTD) method was used to simulate a semirigid base road model with different degrees of looseness. Then, the GPR signal obtained from the forward simulation was numerically counted to extract the attributes related to the extent of looseness of the base course in the simulation signal, which was used to obtain the characteristics for identification of Eigenvalue parameters related to the extent of looseness of the base course. Then, the backpropagation (BP) neural network identification method was used to classify and identify the loose damage of the base course, and the degree of loose damage in the base course was evaluated. This method can reduce the dependence of interpretation of GPR detection images on empirical judgment and provide a new method for the use of GPR to detect the presence and degree of loose damage.

\section{FDTD-Based Forward Simulation of Loose Damage of the Base}

2.1. Road Structure Model. Based on the design scheme of asphalt concrete pavement, the forward model is composed of four layers: the asphalt pavement, semirigid base, subbase, and earth subgrade layer. The length of the model is $2 \mathrm{~m}$ and the depth is $1 \mathrm{~m}$. The dimensions of each road structural layer are shown in Figure $1 ; \varepsilon_{r}$ is the dielectric constant, and $\sigma$ is the conductivity. The surface layer is $0.18 \mathrm{~m}$ deep, the base is $0.38 \mathrm{~m}$ deep, the subbase is $0.2 \mathrm{~m}$ deep, and the soil foundation is below.

Cement-stabilized macadam is one of the most commonly used materials for semirigid base courses. It is a composite material whose dielectric properties are closely related to its composition. According to the volume mixing dielectric model of cement mixtures, the dielectric constant and mention rate of each component of the cement mixture are the main factors affecting the dielectric properties of the material. In the composition of the cement mixture, the higher the dielectric constant of each component material, the larger the volume occupancy rate and the greater the dielectric property of the composite material. The following equation is the root mean square model expression for the dielectric constant of the cement concrete three-phase composite material:

$$
\varepsilon_{r}=\left(V_{a}\left(\varepsilon_{a}\right)^{1 / 3}+V_{b}\left(\varepsilon_{b}\right)^{1 / 3}+V_{c}\left(\varepsilon_{c}\right)^{1 / 3}\right)^{3},
$$

where $\varepsilon_{r}$ is the dielectric constant of the composites; $\varepsilon_{a}, \varepsilon_{b}$, and $\varepsilon_{c}$ are the dielectric constants of each material; and $V_{a}, V_{b}, V_{c}$ are the volume ratios of each material.

When the proportion of air in the cement-stabilized macadam exceeds the design index, looseness damage will occur in the base course. At this time, the dielectric properties of the damaged parts are different from those of the surrounding materials, which allows the semirigid base to be detected and identified by GPR. In this study, the proportion of air in the base is varied to simulate the change in the degree of looseness of the base and investigate the influence of different degrees of loose damage on the electromagnetic wave numerical simulation.

2.2. FDTD Forward Modeling Theory. The FDTD method is an important numerical method for calculating electromagnetic properties [26, 27]. Compared with the moment method and finite element method, the FDTD method has obvious advantages in terms of accuracy and efficiency. In this study, an FDTD code is written in a MATLAB environment using the FDTD method. A two-dimensional forward simulation of the electromagnetic wave propagation of a semirigid base pavement structure with or without damage is realized, and the influence of loose damage on radar electromagnetic wave propagation is studied.

Assuming that the simulated medium is homogeneous and isotropic, the propagation of high-frequency 


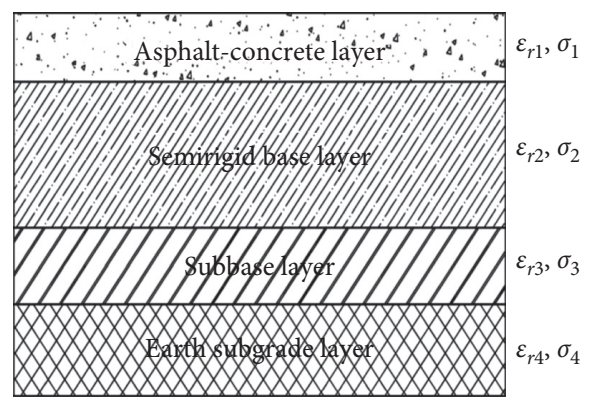

FIGURE 1: Schematic diagram of the pavement structure.

electromagnetic waves in a three-dimensional medium satisfies the following Maxwell equations [28, 29]:

$$
\begin{aligned}
& \nabla \times H=\varepsilon \frac{\partial E}{\partial t}+\sigma E, \\
& \nabla \times E=-\mu \frac{\partial H}{\partial t}-\sigma_{m} H,
\end{aligned}
$$

where $E$ is the electric field intensity $(V / M), H$ is the magnetic field strength $(A / m), \varepsilon$ is the dielectric constant $(\mathrm{F} / \mathrm{m}), \mu$ is the permeability $(\mathrm{H} / \mathrm{m}), \sigma$ is the conductivity $(\mathrm{S} / \mathrm{m})$, and $\sigma_{m}$ is the permeability $(1 / \mathrm{m})$.

In the two-dimensional FDTD scheme, assuming that all of the physical quantities are independent of the $z$-direction, i.e., the two-dimensional expansion becomes an independent transverse magnetic wave (TM) and transverse wave (TE), the following equations can be obtained from equations (2) and (3):

$$
\mathrm{TM}:\left\{\begin{array}{l}
\frac{\partial E_{z}}{\partial y}=-\mu \frac{\partial H_{z}}{\partial t} \\
\frac{\partial E_{z}}{\partial x}=\mu \frac{\partial H_{y}}{\partial t} \\
\frac{\partial H_{y}}{\partial x}-\frac{\partial H_{x}}{\partial y}=\varepsilon \frac{\partial E_{z}}{\partial t}+\sigma E_{x}
\end{array}\right.
$$

$$
\mathrm{TE}:\left\{\begin{array}{c}
-\frac{\partial H_{z}}{\partial x}=\varepsilon \frac{\partial E_{y}}{\partial t}+\sigma E_{y} \\
-\frac{\partial H_{z}}{\partial x}=\varepsilon \frac{\partial E_{x}}{\partial t}+\sigma E_{z} \\
\frac{\partial E_{y}}{\partial x}-\frac{\partial E_{x}}{\partial y}=-\mu \frac{\partial H_{z}}{\partial t} .
\end{array}\right.
$$

Obviously, TM wave only has $E$ component. Since GPR only records $E$ component of horizontal electric field, then we deduce TM wave. Table 1 shows the values of each component of $E$ and $H$ the time step of spatial nodes in the two-dimensional FDTD difference equation.

For equation (3), when $E_{x}=E_{y}=H_{z}=0$, the twodimensional TM wave FDTD equation can be obtained as follows:

$$
\begin{aligned}
H_{x}^{n+1 / 2}\left(i, j+\frac{1}{2}\right) & =H_{x}^{n-1 / 2}\left(i, j+\frac{1}{2}\right)-C P(m) \frac{E_{z}^{n}(i, j+1)-E_{x}^{n}(i, j)}{\Delta y}, \\
H_{y}^{n+1 / 2}\left(i+\frac{1}{2}, j\right) & =H_{y}^{n-1 / 2}\left(i+\frac{1}{2}, j\right)-C P(m) \frac{E_{z}^{n}(i+1, j)-E_{z}^{n}(i, j)}{\Delta x}, \\
E_{z}^{n}(i, j) & =C A(m) E_{z}^{n}(i, j)+C B(m) \times\left[\frac{H_{y}^{n+1 / 2}(i+1 / 2, j)}{\Delta x}-\frac{H_{y}^{n+1 / 2}(i-1 / 2, j)}{\Delta x}-\frac{H_{x}^{n+1 / 2}(i, j+1 / 2)}{\Delta y}+\frac{H_{x}^{n+1 / 2}(i, j-1 / 2)}{\Delta y}\right],
\end{aligned}
$$

where $\quad C A(m)=1-\sigma(m) \Delta t / 2 \varepsilon(m) / 1+\sigma(m) \Delta t / 2 \varepsilon(m)$ $C B(m)=\Delta t / \varepsilon(m) / 1+\sigma(m) \Delta t / 2 \varepsilon(m)$, and $C P(m)=\Delta t /$ $\mu(m)$.

2.3. Forward Modeling. GPR echo signals often have many different characteristics, such as varying frequency, amplitude, and phase. These characteristics reflect the characteristics of echo signals from different aspects and thus reflect the characteristics of underground targets. For loose damage of a semirigid base, because the electromagnetic properties of the damage and its surrounding media are quite different, the echo signal of the damage must differ from that in other places, and its frequency, amplitude, and phase must also have unique properties. The different performance and characteristics of these features reflect the nature of the underground target from different angles, which provides the necessary conditions for echo signal recognition. To study the influence of damage with different extents of looseness on the electromagnetic wave numerical values, the extent of looseness in the base course was varied by adjusting the proportion of air in the base course. The porosity in the loose part was increased by $1 \%$ to a maximum of $30 \%$. A total of 30 loose models were established. A selection of these models and their corresponding forward simulation maps are shown in Figures 2 and 3 .

In this paper, different porosity is used to represent different loose degree of base. In the simulation of models 
TABle 1: Node positions of the TM wave components.

\begin{tabular}{lcccc}
\hline \multirow{2}{*}{ Electromagnetic field component } & \multicolumn{2}{c}{ Spatial component sampling } & $y$ & Time axis $t$ \\
& & $x$ & $j$ & $n$ \\
$\mathrm{TM}$ & $E_{z}$ & $i$ & $j+1 / 2$ & $n+1 / 2$ \\
& $H_{x}$ & $i$ & $j$ & $n+1 / 2$ \\
\hline
\end{tabular}
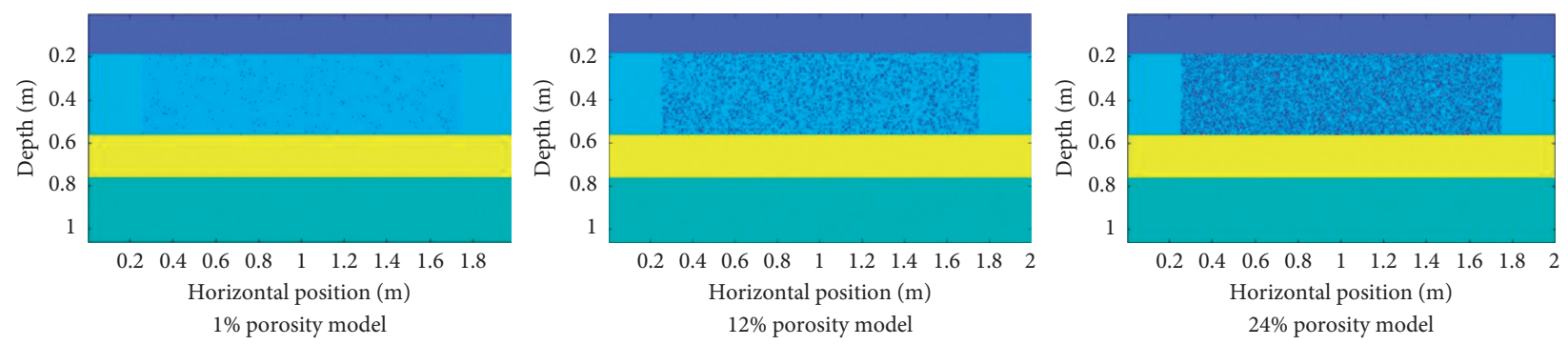

FIgURE 2: Model diagram of different porosity.
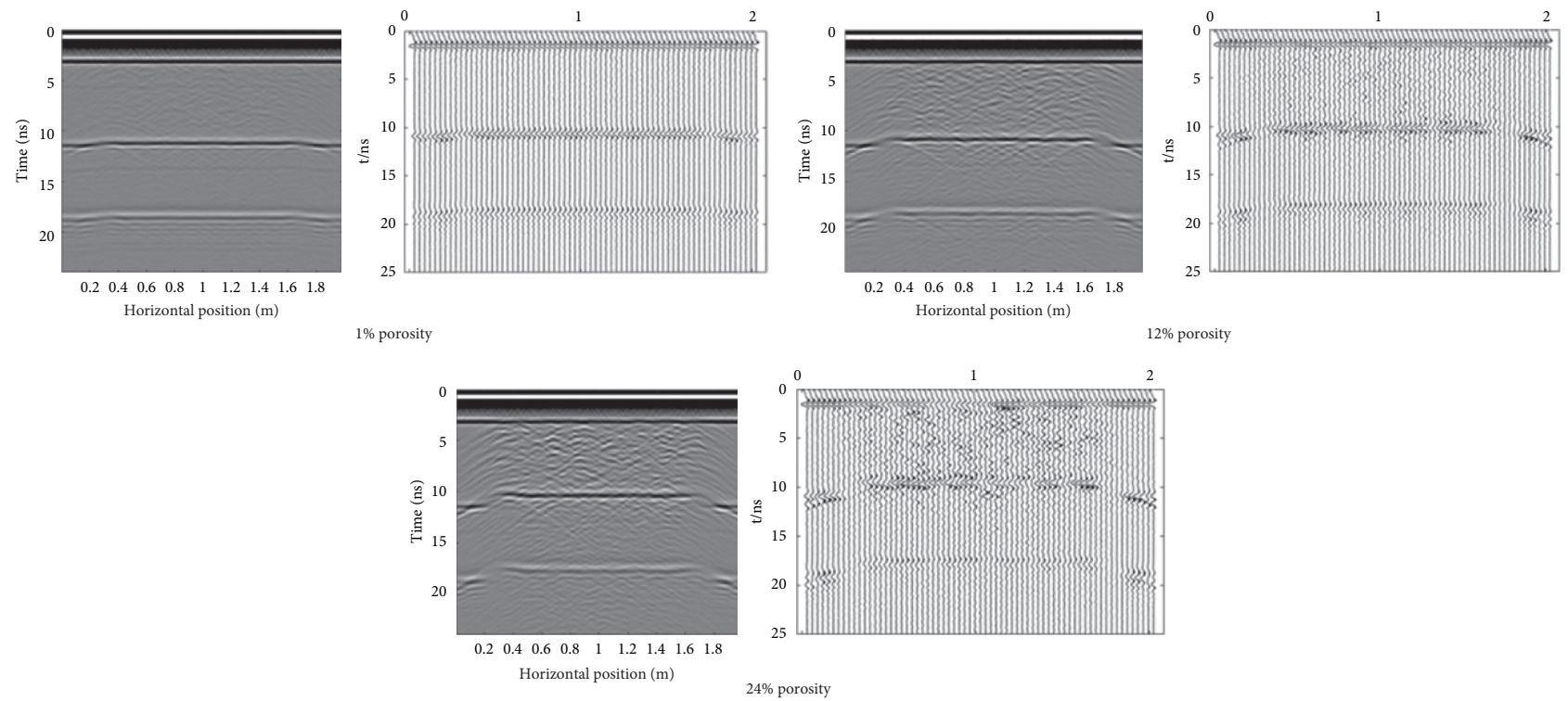

FIgURE 3: Simulation and reflection spectra for different porosity.

with different looseness degrees of the base course, owing to the air dielectric constant being 1, the dielectric constant of base material is between 9 and 12.With an increasing degree of looseness, the proportion of air in base increases gradually, so the composite dielectric constant of base material decreases with the increase of loose degree of base. As a result, the overall dielectric properties of the base material change, and the complexity of the simulation spectra corresponding to different looseness extents is different. When the base is slightly loose, the simulation map exhibits a slight change, but it is roughly consistent with that for the intact road structure. When the extent of the base looseness is severe, the loose damage appears as a discontinuity of the same phase axis, diffraction, and a disordered waveform. According to the relationship between the dielectric properties and electromagnetic wave propagation, different extents of looseness correspond to different electromagnetic wave propagation processes, which provides samples for further classification and recognition.

2.4. Radar Signal Eigenvalue Extraction. Extraction of attributes of the GPR for the base with varying degrees of looseness is a precondition for signal classification and recognition. By preprocessing the identified GPR data, the radar signal features reflecting the extent of looseness of the base course can be extracted. By establishing the relationship between the extent of looseness of the base course and the time-domain attributes of the GPR signals, radar attributes that have a strong correlation with the extent of looseness of the base course can be selected as characteristic values for classification and recognition, and the extent of looseness of the base course can be classified and recognized. Figure 4 shows the radar signal waveforms of different looseness 


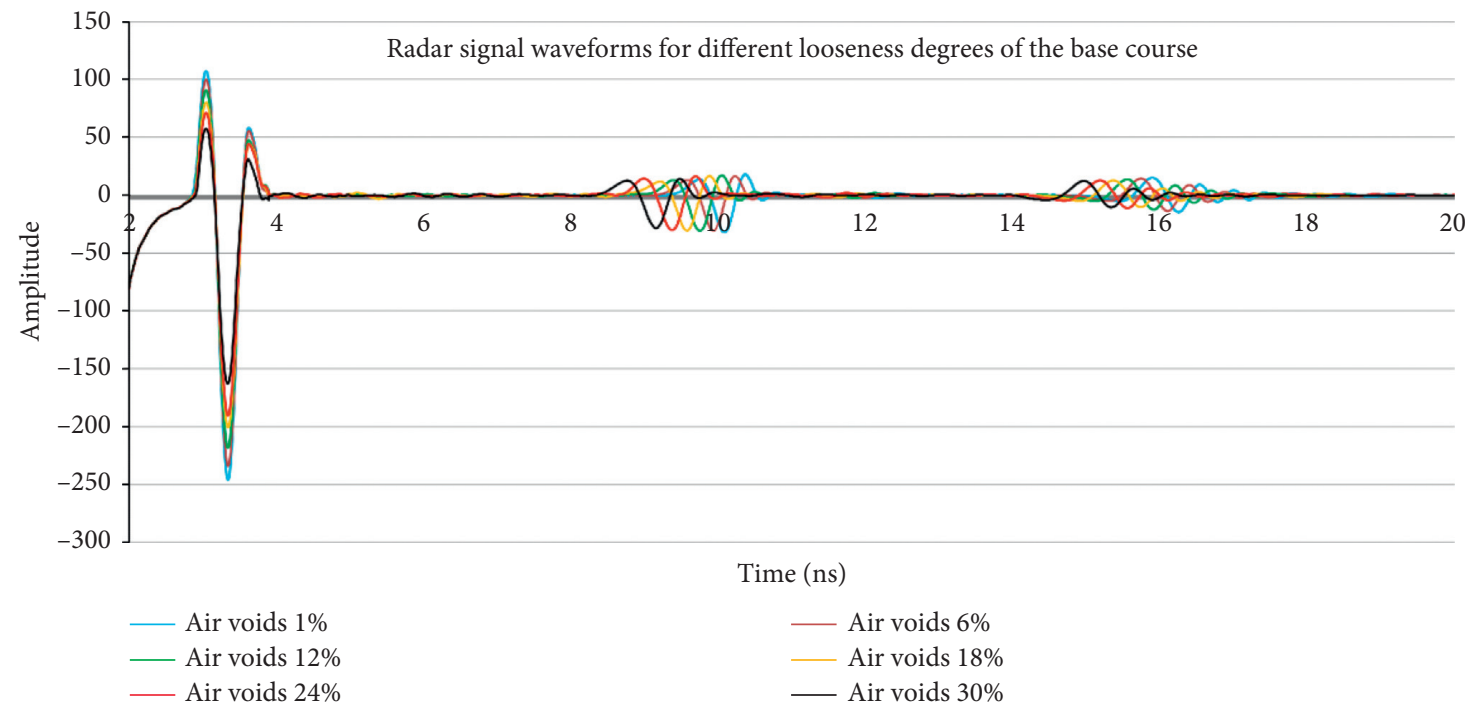

Figure 4: Radar signal waveforms for different looseness degrees of the base course.

degrees in the base course. A time-domain statistical analysis is used to process the GPR signal data, and waveform, amplitude, and other related information of the GPR signal in the time domain are obtained, such as the peak amplitude, trough amplitude, overall average peak amplitude, root mean square amplitude, and other time-domain characteristics.

It can be seen that the corresponding radar data also change with the increase in porosity, i.e., the looseness extent. For the models with different looseness extents, the ground coupling wave remains basically the same. With an increasing extent of looseness of the base, the proportion of air in the base area increases, and the composite dielectric constant of the material in the loose area of the base decreases. Because the dielectric constant of the base material is greater than that of the surface material, the dielectric constant gap between the base and the surface decreases with an increasing extent of looseness of the base course. As a result, the reflected wave amplitude decreases with the increase in looseness. The time delay, $\Delta \mathrm{t}$, decreases with an increasing extent of looseness when the medium thickness is constant.

Through the time-domain attribute analysis of the radar signal after mean processing, nine time-domain eigenvalues of the signal amplitude, such as the average absolute value, variance, root mean square, standard deviation, waveform factor, peak factor, and pulse factor, are shown to have good correlations with the extent of looseness of the base course. As shown in Figure 5, the correlation between the nine eigenvalues and the extent of looseness is good, and thus these can be used for radar signal classification and recognition.

\section{Identification Method for the Looseness Degree of a Semirigid Base Using a BP Neural Network}

Neural networks have good learning, self-adaptive, associative memory, fault-tolerant, classification, and recognition abilities as well as good robustness [30]. BP neural networks have significant advantages in numerical classification and recognition. Therefore, the BP neural network method is selected to realize the classification and recognition of the extent of looseness of the base course. The extracted radar signal numerical characteristics related to the extent of looseness of the base course are used as the input to the BP neural network, and the classification of the extent of looseness of the base course is ultimately output.

3.1. Sample Training. Among the 18,000 samples obtained (These 18,000 samples are obtained from the electromagnetic wave numerical simulation results in Section 2.3. The simulated radar signals are processed by time-domain analysis and the characteristic values related to the looseness of the base are extracted.), $80 \%$ are used as the training sample set and the remaining $20 \%$ are used as test data. For the training samples of the neural network, each input corresponds to an output. Suppose there is a known training set that contains $h$ training samples, and there are $h$ groups of inputs and outputs. Then one group of samples is $X=\left(x_{1}, x_{2}, \ldots, x_{h}\right), Y=\left(y_{1}, y_{2}, \ldots, y_{h}\right)$; the number of neural units in the hidden layer is $p$, and the number of neural units in the output layer is $q$.The steps of the BP neural network algorithm are as follows:

(1) Network Initialization. The weights of each link are assigned to random numbers with intervals $(-1,1)$, and the calculation precision and training times are given.

(2) Determination of the Training Dataset. Some training samples are listed in Table 2. A set of samples, $X=\left(x_{1}, x_{2}, \ldots, x_{h}\right)$, are entered, and the corresponding $Y=\left(y_{1}, y_{2}, \ldots, y_{h}\right)$ is provided to the neural network.

(3) Forward Propagation of the Working Signal. Suppose $X_{i}^{k}$ is the sum of inputs of neuron $i$ in layer $k$, then $Y_{i}^{k}$ is the output, and the weights from the layer $k-1$ 

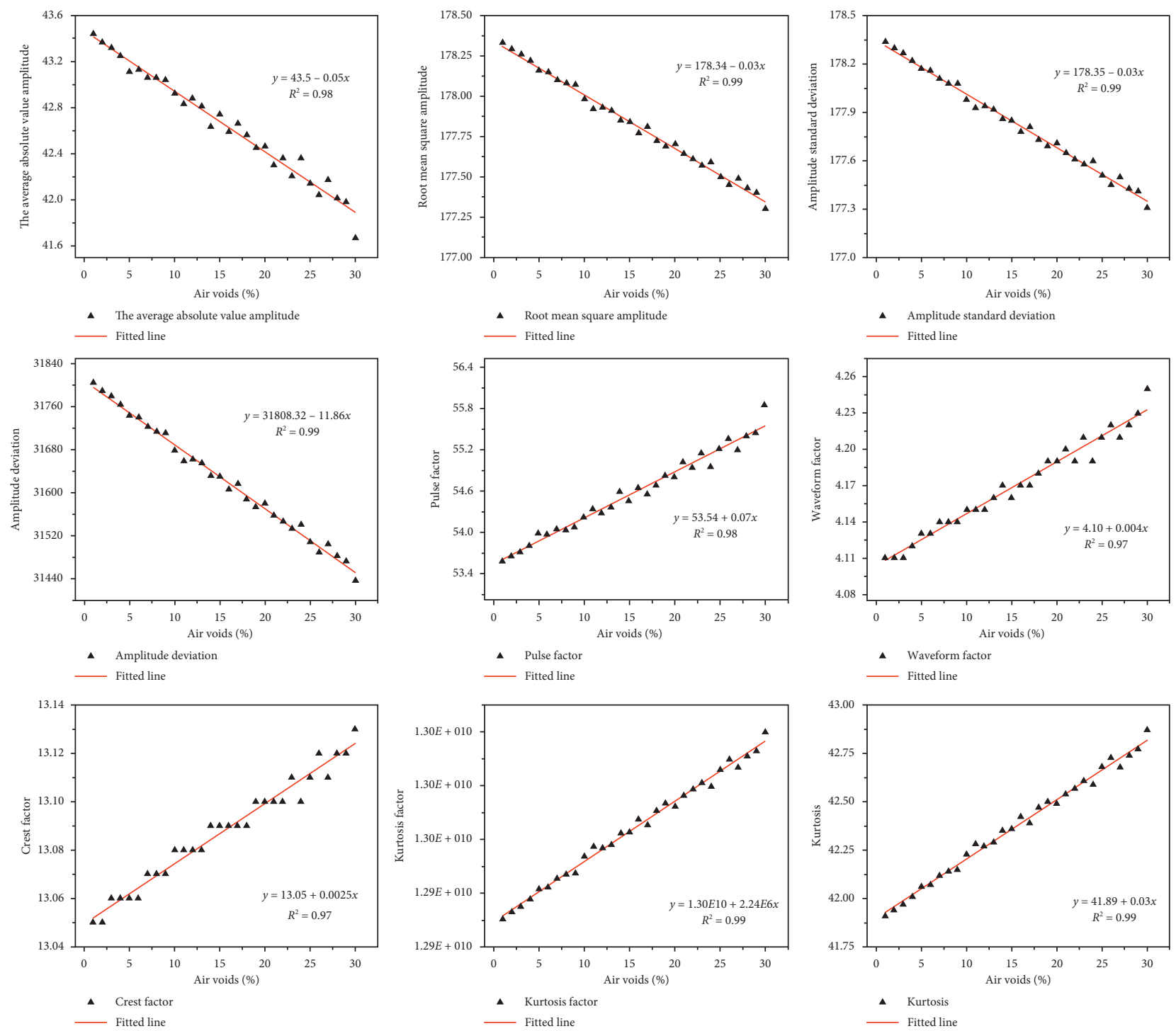

Figure 5: Broken line diagrams of the radar signal time-domain eigenvalues versus the looseness degree.

neurons, $j$, to layer $k$ neurons, $i$, are $w_{i j}$ and have the following functional relationship:

$$
\left\{\begin{array}{l}
Y_{i}^{k}=f\left(X_{i}^{k}\right), \\
X_{i}^{k}=\sum_{j=1}^{n+1} w_{i j} Y_{j}^{k-1},
\end{array}\right.
$$

where $f$ is the activation function. Generally, $f$ is taken as an asymmetric sigmoid function as follows:

$$
f\left(X_{i}^{k}\right)=\frac{1}{1+\exp \left(-X_{i}^{k}\right)} .
$$

(4) Backpropagation of the Error Signal. If the output layer is layer $m$, the actual output of the $i$ th neuron in the output layer is $Y_{i}^{m}$, and the corresponding learning signal is $Y_{i}$. The error function, $e$, is defined as follows:

$$
e=\frac{1}{2} \sum_{i}\left(Y_{i}^{m}-Y_{i}\right)^{2} .
$$

The definition of $d_{i}^{k}$ describes the error function, $e$, with respect to the partial derivative of $Y_{i}^{m}$. Thus, the following can be inferred:

$$
\text { when } \begin{aligned}
k & =m, \\
d_{i}^{m} & =Y_{i}^{m}\left(1-Y_{i}^{m}\right)\left(Y_{i}^{m}-Y_{i}\right), \\
k & <m, \\
d_{i}^{k} & =Y_{i}^{k}\left(1-Y_{i}^{k}\right) \sum_{1} w_{1 i} d_{1}^{k+1} .
\end{aligned}
$$

(5) Correction of the Weights. Let the weight of a certain learning be modified as $\Delta w_{i j}$. Then, considering the correlation between the two learning tasks, the weight modification can be defined as follows: 
TABLe 2: Part of the training sample set.

\begin{tabular}{|c|c|c|c|c|c|c|c|c|c|}
\hline \multicolumn{10}{|c|}{ Attributes } \\
\hline Sample & $\begin{array}{c}\text { Average } \\
\text { absolute value } \\
\text { amplitude }\end{array}$ & $\begin{array}{l}\text { Root mean } \\
\text { square } \\
\text { amplitude }\end{array}$ & $\begin{array}{l}\text { Amplitude } \\
\text { standard } \\
\text { deviation }\end{array}$ & $\begin{array}{c}\text { Amplitude } \\
\text { deviation }\end{array}$ & $\begin{array}{l}\text { Pulse } \\
\text { factor }\end{array}$ & $\begin{array}{l}\text { Waveform } \\
\text { factor }\end{array}$ & $\begin{array}{l}\text { Crest } \\
\text { factor }\end{array}$ & $\begin{array}{c}\text { Kurtosis } \\
\text { factor }\end{array}$ & Kurtosis \\
\hline 1 & 45.83 & 178.68 & 177.65 & 31656.78 & 4.33 & 12.84 & $1.30 E+10$ & 55.73 & 43.04 \\
\hline 2 & 41.38 & 174.03 & 177.19 & 31568.74 & 4.23 & 12.92 & $1.29 E+10$ & 56.04 & 44.90 \\
\hline 3 & 38.21 & 176.94 & 175.61 & 31650.92 & 4.15 & 12.93 & $1.30 E+10$ & 57.22 & 42.39 \\
\hline 4 & 41.29 & 176.45 & 176.54 & 31425.52 & 4.28 & 12.54 & $1.29 E+10$ & 54.66 & 41.58 \\
\hline 5 & 39.49 & 177.28 & 179.34 & 31338.37 & 3.86 & 13.13 & $1.30 E+10$ & 54.01 & 40.45 \\
\hline 6 & 42.46 & 178.08 & 178.72 & 31815.35 & 4.31 & 12.77 & $1.30 E+10$ & 51.66 & 40.96 \\
\hline 7 & 43.04 & 174.59 & 177.12 & 31508.84 & 4.13 & 13.35 & $1.30 E+10$ & 54.16 & 41.12 \\
\hline 8 & 42.05 & 178.87 & 175.06 & 31373.60 & 3.86 & 12.56 & $1.29 E+10$ & 51.19 & 43.30 \\
\hline 9 & 39.89 & 176.52 & 175.89 & 31836.04 & 4.38 & 12.90 & $1.29 E+10$ & 56.91 & 40.16 \\
\hline 10 & 45.72 & 177.35 & 175.16 & 31386.90 & 3.97 & 13.18 & $1.30 E+10$ & 54.73 & 41.29 \\
\hline
\end{tabular}

$$
\nabla w_{i j}(t+1)=\nabla w_{i j}(t)-v \cdot U_{i j}
$$

where $\nabla w_{i j}(t+1)$ and $\nabla w_{i j}(t)$ are the weights in neuron $i$ of layer $j$ after $(t+1)$ th and $(t)$ th epoch; $v$ is learning rate; $U_{i j}$ is the derivative of error $e$ w.r.t. $w_{i j}$ in $(t+1)$ th epoch, which is the same as $d_{i}^{k}$ of Eqs.

(6) The iteration, $E$, is calculated to determine whether the training target is achieved. If the target has been reached or the iteration times have reached the upper limit, the calculation is stopped. If the target is not reached, the process returns to Step 2 for cycle execution. After training, the neural network can be tested using the test sample set, and the grid parameters can be modified continuously according to the test results.

3.2. Test Results. The 30 looseness extents considered are divided into five looseness types, which is convenient for classification and recognition using the neural network. Table 3 lists the classifications of the extent of looseness.

A total of 25 data points were selected as a group of test samples, and 720 tests were carried out; the accuracy was greater than $90 \%$. The feasibility of the classification and recognition method for the extent of looseness of the base course was thus verified. Table 4 summarizes the results of one of the tests. The results include the number of samples, actual category of samples, and type of judgment. At the same time, correct and incorrect judgments are also given. It can be seen from the test results that there were three identification errors, but the judgment categories were similar to the actual categories, and there were no identification errors with significant differences.

\section{Engineering Case Verification}

4.1. Project Overview. To further verify the feasibility of the BP neural network based on GPR technology for the classification and recognition of loose damage in the base course, detection of a road in Zhengzhou city is analyzed using radar detection software in this study. The road
TABLE 3: Classification of the extent of looseness.

\begin{tabular}{cccccc}
\hline Air voids & $\leq 6 \%$ & $6 \%-12 \%$ & $12 \%-18 \%$ & $18 \%-24 \%$ & $\geq 24 \%$ \\
\hline Category & I & II & III & IV & V \\
\hline
\end{tabular}

structure is shown in Table 5. The ground-penetrating radar equipment and road site photos are shown in Figure 6.

The two-dimensional GPR used in this inspection is the GER series from the Qingdao Zhongdian Zhongyi Company. To ensure the accuracy of the detection results, it is necessary to set the antenna center frequency, measuring point spacing, sampling time window, and other measurement parameters before operating the GPR. The frequency of the GPR center antenna is determined based on the measured target depth. In general, the resolution increases with an increase in the radar antenna frequency, while the detection depth decreases. To ensure that the GPR can satisfy the detection requirements, the antenna center frequency should be reduced as much as possible while still meeting the resolution requirements to obtain the radar reflection signal at a deeper position. The maximum detection range of the GPR is $3.0 \mathrm{~m}$, and thus the center antenna frequency is $400 \mathrm{MHz}$, and a time window of $80 \mathrm{~ns}$ is used for detection with a 3-5-point gain.

For the detection of standard sections, the survey lines are arranged along the direction of the carriageway parallel to the centerline of the lane; the influence of terrain and other interference sources is avoided as much as possible. For the detection of abnormal parts, the survey lines can be appropriately encrypted or arranged in a network. When hidden damage is detected under the road surface, two types of antennas with different frequencies should be employed simultaneously for testing to ensure accurate identification of the damaged parts. The layout of the survey lines is shown in Figure 7.

4.2. Analysis and Verification of the Ground-Penetrating Radar Test Data. Through static correction, background filtering, gain adjustment, interference wave elimination, time-depth conversion, and data visualization processing of the GPR data files, a detection Atlas of the GPR is obtained. For the areas with loose damage in the road base, core drilling sampling is carried out to determine the degree of looseness, and radar data are obtained in combination with 
TABle 4: Test results.

\begin{tabular}{|c|c|c|c|c|}
\hline Sample number & Actual category & Judgment category & True/false & Air voids \\
\hline 1 & I & I & True & $<6 \%$ \\
\hline 2 & I & I & True & $<6 \%$ \\
\hline 3 & I & I & True & $<6 \%$ \\
\hline 4 & I & I & True & $<6 \%$ \\
\hline 5 & I & I & True & $<6 \%$ \\
\hline 6 & II & II & True & $6 \%-12 \%$ \\
\hline 7 & II & II & True & $6 \%-12 \%$ \\
\hline 8 & II & II & True & $6 \%-12 \%$ \\
\hline 9 & II & II & True & $6 \%-12 \%$ \\
\hline 10 & II & III & False & $12 \%-18 \%$ \\
\hline 11 & III & III & True & $12 \%-18 \%$ \\
\hline 12 & III & III & True & $12 \%-18 \%$ \\
\hline 13 & III & III & True & $12 \%-18 \%$ \\
\hline 14 & III & III & True & $12 \%-18 \%$ \\
\hline 15 & III & III & True & $12 \%-18 \%$ \\
\hline 16 & IV & IV & True & $18 \%-24 \%$ \\
\hline 17 & IV & IV & True & $18 \%-24 \%$ \\
\hline 18 & IV & III & False & $12 \%-18 \%$ \\
\hline 19 & IV & IV & True & $18 \%-24 \%$ \\
\hline 20 & IV & IV & True & $18 \%-24 \%$ \\
\hline 21 & $\mathrm{~V}$ & $\mathrm{~V}$ & True & $>24 \%$ \\
\hline 22 & V & $\mathrm{V}$ & True & $>24 \%$ \\
\hline 23 & $\mathrm{~V}$ & $\mathrm{~V}$ & True & $>24 \%$ \\
\hline 24 & $\mathrm{~V}$ & $\mathrm{~V}$ & True & $>24 \%$ \\
\hline 25 & V & IV & False & $18 \%-24 \%$ \\
\hline
\end{tabular}

TABLE 5: Road structure drawing.

\begin{tabular}{lccc}
\hline Name of structural layer & Depth $(\mathrm{cm})$ & Dielectric constant & Conductivity (mS/m) \\
\hline AC-13C & 4 & $4-9$ & 0.005 \\
AC-20C & 6 & & 0.05 \\
ATB-25 & 12 & $9-14$ & 0.05 \\
Cement-stabilized macadam & 34 & $9-14$ & \\
Low-dose cement-stabilized macadam & 16 & & 05 \\
\hline
\end{tabular}
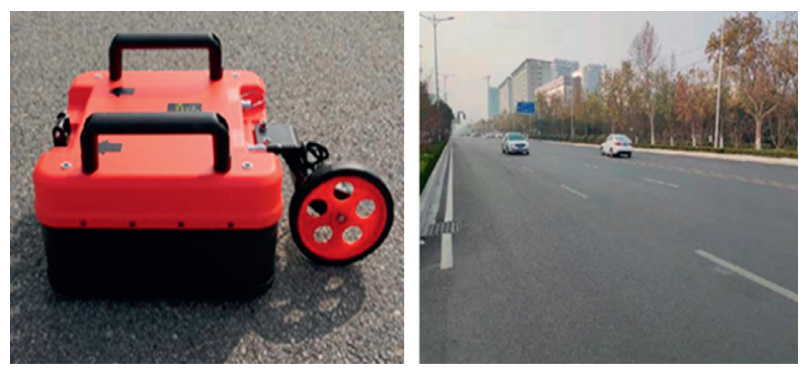

FIgURE 6: Road site photos.

data extraction software. The loose damage types are divided into categories of no loose damage, moderate loose damage, and severe loose damage. Representative GPR detection maps are shown in Figures 8 and 9 .

It can be seen from the actual radar maps in Figures 8 and 9 that the propagation of electromagnetic waves is affected by the loose road structure. Owing to the different degrees of looseness, the radar data will change to varying extents. Comparing the actual maps of severe loose damage and moderate loose damage, the radar-measured map of

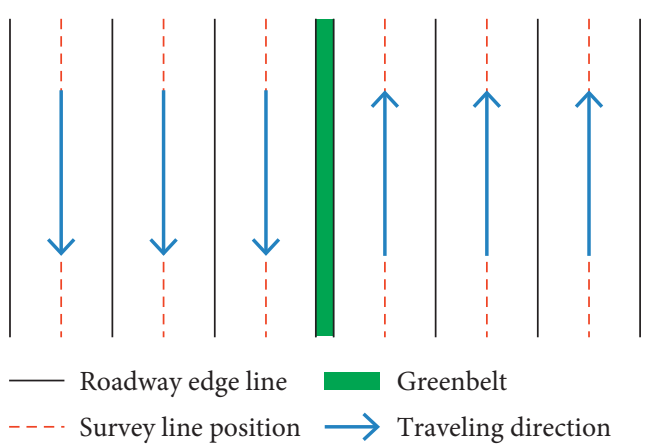

FIgURE 7: Layout of the survey lines.

severe loose damage exhibits a more complex electromagnetic wave pattern. In the actual road, the reflection of the loose area is disorderly, with multiple reflected waves superimposed. In addition, there is water in the base layer of the loose area, which significantly alters the wave amplitude. Based on the response of radar waves to different loose damage degrees, a BP neural network is used to classify and identify the radar data. 


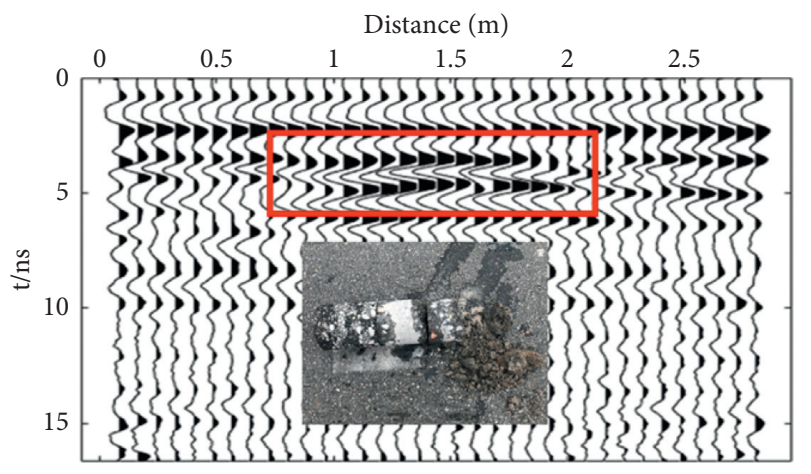

Figure 8: Radar reflection spectrum of moderate loose damage.

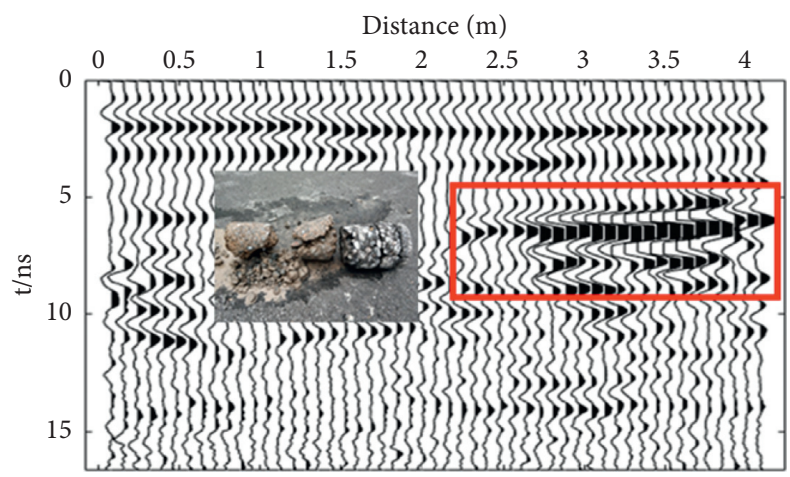

Figure 9: Radar reflection spectrum of severe loose damage.

TABle 6: Classification of the extent of looseness.

\begin{tabular}{lcccc}
\hline Extent of looseness & Normal & Moderate & \\
\hline Category & I & II & Severe \\
\hline
\end{tabular}

TABLe 7: Part of the training sample set.

\begin{tabular}{lccccccccc}
\hline Sample & \multicolumn{1}{c}{ Average absolute } \\
value amplitude & $\begin{array}{c}\text { Root mean } \\
\text { square } \\
\text { amplitude }\end{array}$ & $\begin{array}{c}\text { Amplitude } \\
\text { standard } \\
\text { deviation }\end{array}$ & $\begin{array}{c}\text { Amplitude } \\
\text { deviation }\end{array}$ & $\begin{array}{c}\text { Waveform } \\
\text { factor }\end{array}$ & $\begin{array}{c}\text { Crest } \\
\text { factor }\end{array}$ & $\begin{array}{c}\text { Kurtosis } \\
\text { factor }\end{array}$ & $\begin{array}{c}\text { Pulse } \\
\text { factor }\end{array}$ & Kurtosis \\
\hline 1 & 453.18 & 540.09 & 540.62 & 292271.33 & 1.40 & 8.33 & $3.17 E+10$ & 11.67 & 8.91 \\
2 & 409.97 & 533.14 & 533.66 & 284795.67 & 1.40 & 8.29 & $3.08 E+10$ & 11.58 & 8.99 \\
3 & 439.65 & 513.54 & 514.05 & 264244.13 & 1.40 & 8.49 & $2.97 E+10$ & 11.89 & 9.72 \\
4 & 448.10 & 514.72 & 515.23 & 265457.96 & 1.39 & 8.27 & $2.77 E+10$ & 11.45 & 9.00 \\
5 & 376.87 & 503.89 & 504.38 & 254402.69 & 1.38 & 8.28 & $2.58 E+10$ & 11.45 & 8.93 \\
6 & 394.37 & 479.01 & 479.48 & 229899.25 & 1.40 & 8.49 & $2.41 E+10$ & 11.87 & 9.71 \\
7 & 391.61 & 471.10 & 471.56 & 222370.98 & 1.40 & 8.53 & $2.27 E+10$ & 11.96 & 9.59 \\
8 & 355.54 & 462.48 & 462.94 & 214309.79 & 1.41 & 8.65 & $2.2 E+10$ & 12.17 & 9.86 \\
9 & 340.09 & 459.21 & 459.66 & 211290.84 & 1.41 & 8.76 & $2.18 E+10$ & 12.34 & 9.96 \\
10 & 373.81 & 464.46 & 464.91 & 216141.95 & 1.42 & 8.82 & $2.23 E+10$ & 12.52 & 9.86 \\
\hline
\end{tabular}

According to the characteristic values of the radar data, a reasonable BP neural network structure is designed to identify the types of loose damage. The characteristic values of the radar data and corresponding degree of looseness are obtained as BP neural network training samples. Of the sample data, $80 \%$ are used as training samples, and the remaining $20 \%$ are used as test samples to train and test the
BP neural network. Table 6 presents the looseness degree classification table, and Table 7 lists some sample data.

The trained neural network was tested using the measured radar data. The 150 channel-measured radar data were divided into 10 groups with 15 channels in each group, and 10 tests were conducted. Compared with the results of core drilling and sampling, the accuracy of the neural network 
TABLE 8: Statistical analysis of test results.

\begin{tabular}{llllllllll}
\hline Test group & 1 & 2 & 3 & 4 & 5 & 6 & 7 & 8 & 9 \\
\hline Accuracy (\%) & 86 & 93 & 80 & 93 & 80 & 93 & 80 & 87 & 80 \\
\hline
\end{tabular}

TABLE 9: Results of the fourth test.

\begin{tabular}{|c|c|c|c|c|}
\hline Sample number & Actual category & Judgment category & True/false & Extent of looseness \\
\hline 1 & $\mathrm{I}$ & I & True & Normal \\
\hline 2 & I & I & True & Normal \\
\hline 3 & I & I & True & Normal \\
\hline 4 & I & I & True & Normal \\
\hline 5 & I & I & True & Normal \\
\hline 6 & II & II & True & Moderate \\
\hline 7 & II & II & True & Moderate \\
\hline 8 & II & II & True & Moderate \\
\hline 9 & II & II & True & Moderate \\
\hline 10 & II & III & False & Severe \\
\hline 11 & III & III & True & Severe \\
\hline 12 & III & III & True & Severe \\
\hline 13 & III & III & True & Severe \\
\hline 14 & III & III & True & Severe \\
\hline 15 & III & III & True & Severe \\
\hline
\end{tabular}

was greater than $80 \%$. Tables 8 and 9 summarize the statistical analysis of the 10 test results and present the results of the fourth test, respectively.

After testing the trained neural network numerous times, the comprehensive accuracy of the test was greater than $80 \%$, which further verifies the feasibility of the BP neural network for the classification and recognition of the looseness degree in a semirigid base. However, the accuracy of the test results using the actual detection data was slightly lower than the recognition accuracy of the simulation data. The reason is that the numerical simulation situation is relatively ideal, whereas in the actual project, the situation is more complex and the material distribution is uneven. GPR data are affected by the surrounding environment and the acquisition system, and thus it is difficult to reflect the real situation of the underground target accurately, resulting in relatively large error in the identification process.

\section{Conclusions}

To solve the problem that loose damage of a road base cannot be identified quantitatively, and thus the classification and identification of the extent of looseness mainly depends on experienced judgment, this study establishes a structural model of a semirigid base asphalt pavement with different extents of looseness. The FDTD method is then used to simulate the road model with different extents of looseness, and the extent of looseness in the base is extracted using a time-domain analysis method. Then, a BP neural network is used to classify and identify loose damage in the base. The conclusions of this study are as follows:

(1) In the simulated radar echo signal, the average absolute value, root mean square, standard deviation, waveform factor, peak factor, pulse factor, and other time-domain eigenvalues are shown to have a good correlation with the extent of looseness of the base, allowing these values to be used as the basis for radar signal classification and recognition.

(2) With increasing looseness of the base, the corresponding simulation map becomes more complex, and the reflected amplitude decreases with increasing looseness degree. The time delay, $\Delta t$, decreases with an increasing extent of looseness when the medium thickness is constant.

(3) The recognition accuracy of the simulated electromagnetic wave is greater than $90 \%$. The radar data of the actual project are classified and identified, and the recognition accuracy is greater than $80 \%$. The results of the classification and recognition of the simulated data and measured data verify the feasibility of the classification and recognition method, which can provide a new method for the use of GPR to detect the presence and degree of loose damage.

\section{Data Availability}

The data used to support the findings of this study are included within the article.

\section{Conflicts of Interest}

The authors declare that they have no conflicts of interest regarding the publication of this paper.

\section{Acknowledgments}

The authors acknowledge the support received from the National Key Research and Development Plan (Grant no. 2018YFB1600200), the National Natural Science Foundation of China (Grant nos. 51878624 and 51878622), Major Scientific and Technological Special Project in Henan (Grant no. 181100310400), Henan Science Fund for Distinguished 
Young Scholars (Grant no. 202300410354), Natural Science Foundation of Henan Province (Grant no. 202300410746), the Program for Science and Technology Innovation Talents in Universities of Henan Province (Grant no. 19HASTIT041), and Key Research Projects of Higher Education in Henan Province (Grant no. 18A580001).

\section{References}

[1] S. H. A. Ai-min, "Material characteristics of semi-rigid base," China Journal of Highway and Transport, vol. 21, no. 001, pp. 1-5, 2008.

[2] C. Jing, J. Zhang, and B. Song, "An innovative evaluation method for performance of in-service asphalt pavement with semi-rigid base," Construction and Building Materials, vol. 235 , p. 117376, 2020.

[3] X. Wang and X. Ma, "Responses of semi-rigid base asphalt pavement with interlayer contact bonding model," Advances in Civil Engineering, vol. 2020, no. 3, pp. 1-13, 2020.

[4] H. Ahn and J. Lee, "Subsurface condition evaluation of asphalt pavement for pavement preservation treatments," Journal of Testing and Evaluation, vol. 44, no. 3, pp. 1183-1193, 2016.

[5] Y. A. N. G. Yong, "The influence of factors on the durability of asphalt pavement," International Journal of Civil Engineering and Machinery Manufacture, vol. 2, no. 2, 2017.

[6] Y. Wen, G. Hong, "Research on crack-resistance property of asphalt mixture as road stress-absorbing interlayer," $A d$ vanced Materials Research, p. 1333, 2011.

[7] V. . Marecos, S. Fontul, M. D. L. Antunes et al., "Evaluation of a highway pavement using non-destructive tests: falling weight deflectometer and ground penetrating radar," Construction and Building Materials, p. 154, 2017.

[8] D. Zehua, Y. Shengbo, G. Yunze et al., "Rapid detection methods for asphalt pavement thicknesses and defects by a vehicle-mounted ground penetrating radar (GPR) system," Sensors (Basel, Switzerland), vol. 16, no. 12, 2016.

[9] S. Wang, S. Zhao, I. L. Al-Qadi, and Al-Qadi, "Continuous real-time monitoring of flexible pavement layer density and thickness using ground penetrating radar," NDT \& E International, vol. 100, pp. 48-54, 2018.

[10] V. Sunjay and V. K. Singh, "Ground penetrating radar (GPR) applications in hydrogeological study of aquifers," Earth Science Frontiers, vol. 16, no. S1, p. 28, 2009.

[11] J. Gao, D. Yuan, Z. Tong, J. Yang, and D. Yu, "Autonomous pavement distress detection using ground penetrating radar and region-based deep learning," Measurement, vol. 164, p. 108077, 2020.

[12] S. Zhang, W. Shen, D. Li et al., "Nondestructive ultrasonic testing in rod structure with a novel numerical Laplace based wavelet finite element method," Lat. Am. J. Solids Struct, vol. 15, no. 7, 2018.

[13] M. V. Felice and Z. Fan, "Sizing of flaws using ultrasonic bulk wave testing: a review," Ultrasonics, Ultrasonics, vol. 88, pp. 26-42, 2018.

[14] V. Vyas, V. J. Patil, A. P. Singh et al., "Application of infrared thermography for debonding detection in asphalt pavements," Journal of Civil Structural Health Monitoring, no. 29, 2019.

[15] K. Chahine, V. Baltazart, X. Dérobert et al., "Blind deconvolution via independent component analysis for thin-pavement thickness estimation using GPR[C]//2009 International Radar Conference Surveillance for a Safer World," IEEE, (RADAR 2009), 2010.
[16] E. Cardarelli, C. Marrone, and L. Orlando, "Evaluation of tunnel stability using integrated geophysical methods," Journal of Applied Geophysics, vol. 52, no. 2-3, pp. 93-102, 2003.

[17] A. Karem, A. B. Khalifa, and H. Frigui, "A Fisher vector representation of GPR data for detecting buried objects SPIE Defense + Security," Detection and Sensing of Mines Explosive Objects, and Obscured Targets, vol. XXI, 2016.

[18] Gao, Jie, Zhang et al., "Recognition, location, measurement, and $3 \mathrm{D}$ reconstruction of concealed cracks using convolutional neural networks," Construction and Building Materials, 2017.

[19] TongG. Zheng et al., "Innovative method for recognizing subgrade defects based on a convolutional neural network," CONSTRUCTION AND BUILDING MATERIALS, 2018.

[20] A. Cubero-Fernandez, F. J. Rodriguez-Lozano, R. Villatoro, J. Olivares, and J. M. Palomares, "Efficient pavement crack detection and classification," Eurasip Journal on Image \& Video Processing, vol. 2017, no. 1, 2017.

[21] S. Solla, Approach to Identify Cracking in Asphalt Pavement Using GPR and Infrared Thermographic Methods: Preliminary findings, Ndt \& E International, 2014.

[22] R. DITOMMASO, M. MUCCIARELLI, and F. C. PONZO, "Stransform based filter applied to the analysis of non-linear dynamic behavior of soil and buildings," Pro-ceedings of European Conference on Earthquake Engi-Neering, vol. 30, pp. 1-13, 2010.

[23] X. Xu, Q. Zeng, D. Li et al., "GPR detection of several common subsurface voids inside dikes and dams," Engineering Geology, vol. 111, no. 1-4, pp. 31-42, 2010.

[24] B. Zhang, H. Fang, Y. Zhong et al., "Application of GPR and FWD in comprehensive evaluation of old road performance," Journal of China \& Foreign Highway, vol. 28, no. 005, pp. 89-93, 2008.

[25] B. Zhang and Q. Liu, "Yanhui zhong analysis of dynamic response of rigid pavement deflection under FWD load," Journal of Highway and Transportation Research and Development, vol. 26, no. 7, pp. 33-37, 2009.

[26] T. Meagher, B. Jiang, and P. Jiang, "An enhanced finite difference time domain method for two dimensional Maxwell's equations," Numerical Methods for Partial Differential Equations, vol. 36, no. 5, 2020.

[27] J. Lei, Z. Wang, H. Fang et al., "Analysis of GPR wave propagation in complex underground structures using CUDA-implemented conformal FDTD method," International Journal of Antennas and Propagation, 2019.

[28] K. S. Yee, "Numerical solution of initial boundary value problems involving Maxwell's equations in isotropic media," IEEE Trans. Antennas and Propagation, p. 14, 1966.

[29] Y. Li, Z. Zhao, W. Xu, Z. Liu, and X. Wang, "An effective FDTD model for GPR to detect the material of hard objects buried in tillage soil layer," Soil and Tillage Research, vol. 195, p. 104353, 2019.

[30] X. Li, C. Yuan, and B. Shan, "System identification of neural signal transmission based on backpropagation neural network," Mathematical Problems in Engineering, vol. 2020, no. 1, pp. 1-8, 2020. 